\title{
A SYSTEMATIC COLLECTION OF NATURAL INTERACTIONS FOR IMMERSIVE MODELING FROM BUILDING BLOCKS
}

\author{
Harlan, Jakob; \\ Schleich, Benjamin; \\ Wartzack, Sandro \\ Friedrich-Alexander-Universität Erlangen-Nürnberg
}

\begin{abstract}
The increased availability of affordable virtual reality hardware in the last years boosted research and development of such systems for many fields of application. While extended reality systems are well established for visualization of product data, immersive authoring tools that can create and modify that data are yet to see widespread productive use. Making use of building blocks, we see the possibility that such tools allow quick expression of spatial concepts, even for non-expert users. Optical handtracking technology allows the implementation of this immersive modeling using natural user interfaces. Here the users manipulated the virtual objects with their bare hands. In this work, we present a systematic collection of natural interactions suited for immersive building-block-based modeling systems. The interactions are conceptually described and categorized by the task they fulfil.
\end{abstract}

Keywords: Virtual reality, Conceptual design, Computer Aided Design (CAD)

\section{Contact:}

Harlan, Jakob

Friedrich-Alexander-Universität Erlangen-Nürnberg

KTmfk

Germany

harlan@mfk.fau.de 


\section{INTRODUCTION}

The increased availability of affordable virtual reality (VR) hardware in the last years boosted research and development of such systems for many fields of application. For product design, immersive interfaces hold tremendous potential to improve many tasks in the development process (Coburn et al., 2017). For visualization of geometric and additional product data, extended reality systems are well established, e.g., for design reviews. Immersive authoring tools that can create and modify product data, on the other hand, are yet to see widespread productive use. We see the possibility that such tools allow quick expression of spatial concepts, even for non-expert users. In contrast to the parametric modeling paradigm that prevails in traditional computer-aided design (CAD) software, a more direct, building-block-based modeling paradigm should be used for this use-case (Fechter et al., 2020).

Currently, most VR interfaces rely on controller-based interactions. These provide stable and precise tracking, haptic vibrational feedback, and physical buttons for reliable inputs. But the mapping of the controller's buttons and triggers to commands adds a layer of abstraction between the user's intent and its execution which has to be learned. Natural user interfaces (NUIs) try to minimize this learning by designing interactions that closely mimic real-world actions. Hand tracking technology which continuously captures hand and finger positions in three dimensions allows the implementation of NUIs for immersive systems where users interact with the virtual world using their bare hands. Having a controller- and markerless hand tracking also enables the use of an extended reality (XR) device without further preparation besides putting on a headset. The hands are kept free which allows flexible combination with existing manual interfaces. The interest in bare-hand interactions is not only academic, mayor XR companies offer products using hand-tracking technology as well. Microsoft's HoloLens 2, the Magic Leap One, and the Oculus Quest 2 all have optical hand-tracking build in.

In this work, we present a systematic collection of natural interactions suited for immersive buildingblock-based modeling systems. The interactions are conceptually described and categorized by the task they fulfil. The main novelty and benefit of this paper lies in the provision of catalogue of natural interactions, which supports other researchers in identifying suitable interactions for their immersive modeling systems. For simplicity, state-of-the-art visual hand-tracking and gesture-recognition are assumed. These technologies are large research fields (Rautaray and Agrawal, 2015) and as such are not discussed in this work.

\section{RELATED WORK}

\subsection{Natural modeling in the product design process}

Large parts of the digital engineering design process make use of authoring tools for three-dimensional geometric data. Wherever generation and modification of models are possible we believe intuitive and immersive editing can be beneficial. Applicability peaks in the early geometric design phases, where the rough geometry and effective structure are shaped. Here the ability to quickly express concepts is key, as fast iterations lead to an accelerated solution-finding. The precise interdependent modeling of the detailed design phase is hard to archive with immersive interfaces, but even in that phase immersive modeling might be deployed to enrich for example design reviews.

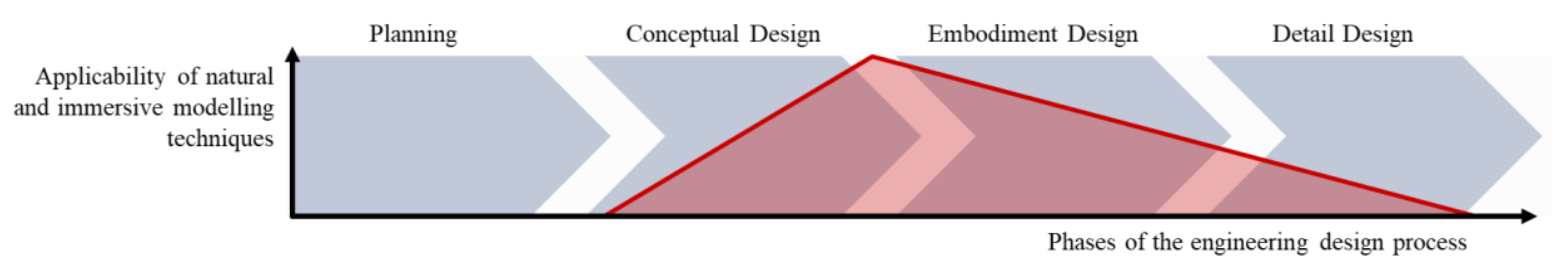

Figure 1. Applicability of natural and immersive modeling techniques in the engineering design process phases based on (Pahl et al., 2013)

\subsection{Research on natural user interfaces in engineering design}

Motivated by the broad applicability of natural virtual reality interfaces in general and by the benefits of such interfaces in engineering design, Regazzoni et al. (2018) formulated some basic guidelines to design natural user interfaces. They have split their guidelines into three categories: dialog principles, guidelines for ergonomics, and gesture guidelines. For this work, the most relevant guidelines are: 
avoiding too complex or lengthy gestures; taking natural real-world interactions as inspiration; limiting the number of gestures the user has to learn; and considering the ergonomics to prevent fatigue.

One way to find natural gestural interactions is to ask users to carry out a task the way they think the interaction should work and record the gestures they do. Doing so in a Wizard of Oz experiment, Cordeiro et al. (2018) researched gestures for three-dimensional shape manipulation and deformation tasks. Vuletic et al. (2018) performed a similar study to identify natural gesture interactions for object manipulation of 3D objects on a 2D screen. The suitability for implementation of resulting gestures has to be analyzed. Recognizability and efficiency are exemplary properties to consider.

In another article, Cordeiro et al. collated around 40 immersive shape manipulation system publications. In their survey, they sorted the systems by their input methods, type of shape manipulation, and shape representations. The collection reaches beyond the scope of this work, but fitting interactions are found under the gesture and hands-free input methods and the CAD model shape representations. One modeling system included in the survey of Cordeiro et al. was presented by Kang et al. (2013) and features the gesture-based design of concepts from simple building blocks. Their modeling paradigm and use case are similar to the systems we envision in this work. The main drawback of their system is the use of a 2D screen, which creates a mismatch between input and output.

\section{COLLECTION OF NATURAL INTERACTIONS}

To identify the central tasks in a body-based modeling tool, we reviewed existing commercial and academic software offering simple three-dimensional modeling capabilities. For this, it does not matter whether they already utilize immersive technologies or bare-handed gestures. For example, the Microsoft 3D Builder (Microsoft, 2013) provides basic modeling features for all Windows 10 users. Other programs we inspected are Sketchup, Oculus Medium, Google Blocks, Gravity Sketch, and Flying Shapes. The essential tasks for building-block-based modeling most programs could execute were collected and summarized in three high-level tasks: Single body creation, assembling the model and non-spatial tasks.

The interactions presented in this work were gathered in multiple ways. One central option was reviewing the existing academic work in this field. The survey from Cordeiro et al. (2019) provided plenty of starting points. Secondly, existing software tools were inspected for this step as well, but as hand-tracking devices are not widespread so is the support for them. The Leap Motion Gallery provides a range of software prototypes for their device, most notably for this work are the Blocks and Button Builder demonstrators. Additionally, interactions developed as part of our research in natural CAD assembly and modeling will be mentioned as well (Fechter et al., 2020; Fechter and Wartzack, 2017; Harlan et al., 2020).

\subsection{Single body creation}

When creating a single body, the first task is to choose its primitive shape. In existing tools, common shapes to choose from are the cuboid, cylinder, and sphere but depending on the field of application the pyramid, cone, or torus might also be included. After a shape is selected the next task is to initialize the body, meaning assigning it its first position and dimensions. The last task associated with the creation of single bodies is the modification of the shape. This can be the simple scaling of the whole body along all or selected axis. But this also includes all kinds of non-transformational operations.

\subsubsection{Shape Selection}

In this task, either the three-dimensional shape or a two-dimensional profile is chosen to be initialized by the following interaction. All interactions for this task follow the pattern recognition over recall by showing the user all options and not requiring him to remember them.

\section{Non-Spatial}

The shape of a body can be chosen before its initialization in a non-spatial manner. Menus and symbolic gestures, interactions further explained in section 3.3, can be used to do so. Menus would show an image, preview rendering, or a labeled button for the user to select the wanted shape. With symbolic gestures, on the other hand, the user has to position its hands and fingers in a way for the computer to infer the desired shape. 

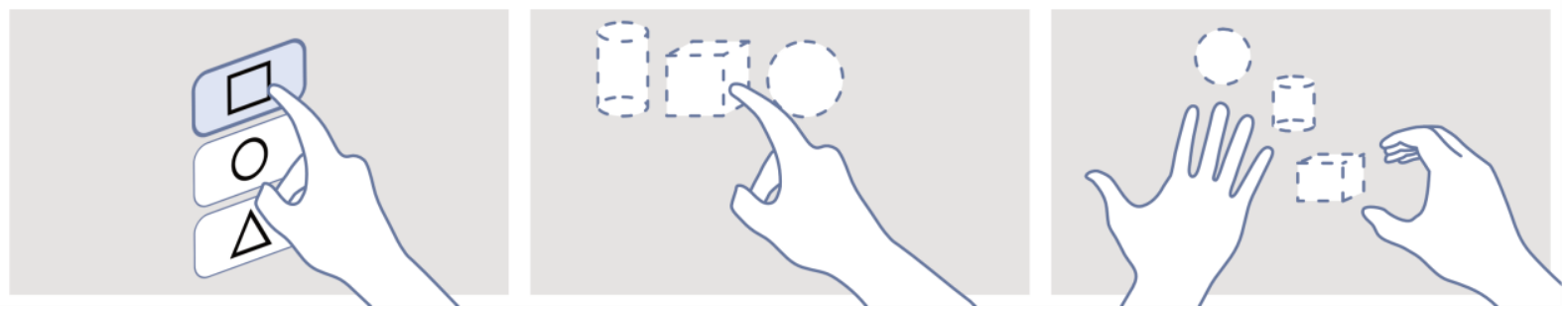

Figure 2. Shape selection interactions: a) non-spatial b) prototypes c) mid-initialization

\section{Prototypes}

For this interaction, instances of all possible shapes are placed in the virtual world for the user to choose from. To avoid confusion the prototypes can be placed in a special area, for instance, a shelf. Alternatively, they might be visualized differently from the created bodies, by showing them transparent, as a wireframe, or with a blueprint-like texture.

\section{Mid-Initialization}

The last presented option is to choose the desired shape while initializing the body. Here, instead of displaying the shapes permanently as prototypes, the shapes are shown at the exact moment the selection has to be made and in a position adapted to the initialization interaction. For example, if the user points the palm of one hand towards him, wireframes of the possible shapes can be distributed around that hand to be picked up by the other hand (Fechter et al., 2020).

\subsubsection{Body initialization}

After the desired shape is selected an initialization, i.e., a first dimensioning and positioning, of the new body is necessary. Coupling shape selection and body initialization can create a fluid body creation interaction.
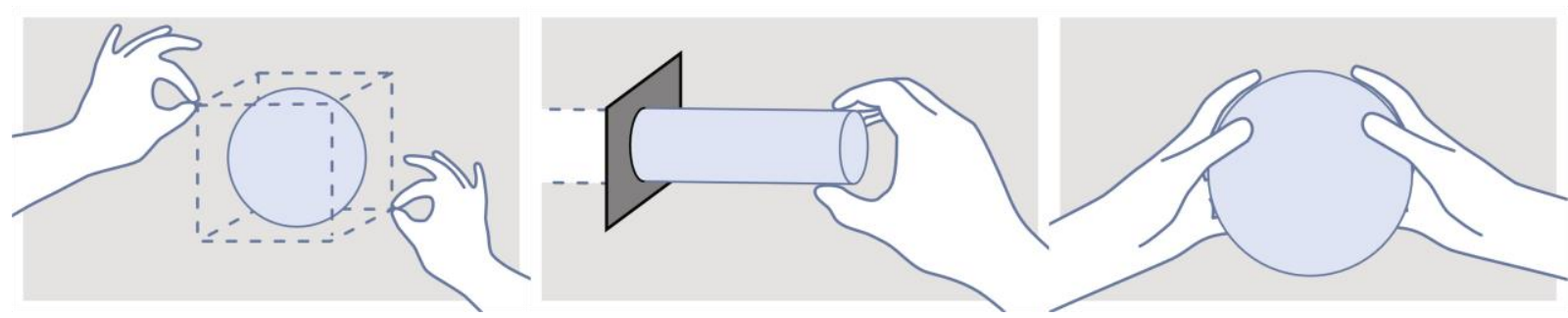

Figure 3. Body initialization interactions: a) two-point pull apart b) extrusion c) summoning

\section{Default generation}

Default generation is the most basic option for body initialization and can be combined with all shape selection interactions. Here, instead of giving the user the possibility to determine the body's initial position and dimensions the body is placed automatically, and shape modifying interactions (Fehler! Verweisquelle konnte nicht gefunden werden.) can then be used to change it. To trigger the default generation a menu button could be pressed, or a prototype grabbed from a shelf. Because the user has no immediate option to adjust the body, useful default sizes determine much of this interaction's usability.

\section{Two-point pull apart}

Making use of both hands, this is an effective interaction to quickly create bodies dimensioned to the user's intent. Two control points grabbed or pinched with each hand, determine the body's dimensions.

The interaction can either set the body's scale in three degrees of freedom (DOF) and leave its rotation fixed or only scale in one direction and adjust the rotation accordingly (Fechter et al., 2020). For this interaction, mid-initialization shape selection is well suited. After pinching with both hands in mid-air, shape proposals could be visualized in between both hands and would be selected by moving both pinched positions close to the desired shape. The two-handed pulling gesture resembles using a measuring tape and while the executed task is different, users might be comfortable with the movements themselves. 


\section{Extrusion}

Extrusion is an interaction conceptually similar to the sketch-and-extrude workflow of many CAD tools. For this, not the 3D shape but a $2 \mathrm{D}$ profile is selected and extended to the required size. An abstract version of this concept would be to use the pull-apart interaction on a wireframe $2 \mathrm{D}$ profile. A more physically motivated and therefore natural version could show the profile as a virtual die and allow the user to pull play-doh-like material through the opening.

\section{Summoning}

Summoning interactions are a playful option for body initialization. Here the system tries to determine the intended primitive and dimensions directly from the hand posture. Holding the hands as if one would hold a ball would create a sphere of that imaginary ball's size, other shapes could be created similarly. While this interaction, resembling e.g., a fictional sorcerer summoning objects, is not truly natural, users could imagine themselves in that position. A confident implementation of this interface, inferring the size, positioning and shape if the user's intended geometry from hand tracking data, will be challenging.

\subsubsection{Shape Modification}

The shape of an already created body should be changeable. For simple bodies replacing an existing one with a different one might be feasible. If, on the other hand, the geometry has increased complexity for example through Boolean operations, redoing the steps for a small change is tedious.
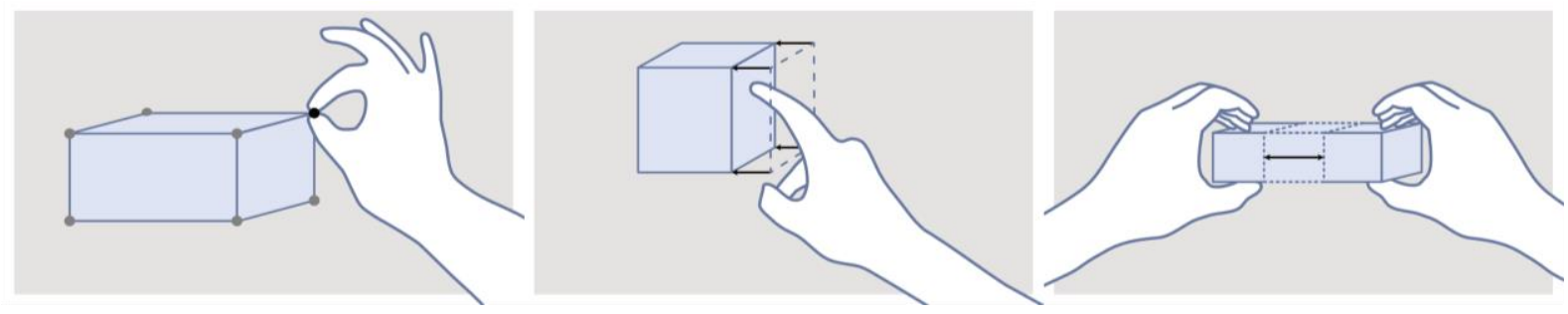

Figure 4. Shape modification interactions: a) control points c) face pushing d) stretching

\section{Control points}

For this interaction, control points are placed on the body to manipulate its shape. To grab these points with good visibility and control, the pinch gesture is well suited. The control points could influence the shape in different ways, one simple option is to control the body's bounding box with the control points and adjust the body accordingly to fit into the box. Control point interactions have increased precision by separating the DOFs and the results are predictable. They are not quite natural, but users of traditional modeling tools will find them intuitive as they are in widespread use.

\section{Face pulling/ pushing}

This interaction allows the faces of a body to be directly modified. This can be achieved both by pulling a face or pushing onto a face. To pull a face the user would pinch very close to it and then pull away from it, stretching the rest of the body accordingly. Pushing can work without a starting gesture like pinching. The faces simply always retract inwards when colliding with the fingers. To form an intuitive interface, the faces need to have different behaviour to the manipulation. While a planar face moves along its normal direction, arced and spherical surfaces increase and decrease their radius. Pulling and pushing can be extended to edges and corners of shapes as well, allowing more flexibility. This direct modification of the geometry creates a natural interaction.

\section{Stretching}

A natural way to change a body's shape is imagining it as an elastic body. This could result in a stretching interaction where grabbing the body at two points allows the user to stretch and compress the body. The interaction would perform a global scaling or scale along one axis. Advanced versions where only the part of a body between the two hands is stretched and parts beyond them are left untouched are imaginable. Even further possibilities are twisting and bending of the grabbed body (Cordeiro et al., 2018).

\subsection{Assembling the model}

To assemble a model from individual bodies, a selection of tasks can be done. The essential task is moving bodies. The movement can be supported by placement aids, which eases positioning the bodies in common configurations. Object selection allows for further common operations including 
coloring, deletion, and copying of bodies. Lastly, a modeling system gets much more powerful by supporting the combination of bodies. The most widespread method are the Boolean operations, allowing two bodies to be united, subtracted, or intersected.

\subsubsection{Moving Bodies}

From virtual reality games to augmented reality maintenance support, in almost all extended reality applications the ability to relocate objects is crucial. Many different interactions have been proposed (Cordeiro et al., 2019) and are often supported by placement aids such as snapping. Below an excerpt is presented, focusing on direct hand-object interactions. Interfaces allowing the movement of an object out of physical reach exist but are omitted here.

\section{Grasping}

The most natural interaction for object relocation is one that everybody does daily, grasping the object. There is long-lasting research interest in realistic virtual grasping, with Christoph Borst being one of the most active authors in this field (Borst and Indugula, 2005). Multiple variants of grasping can be found. Often, to make an interaction more robust to tracking noise, the object's movement is coupled to the palm's transform after a grasp has been detected by collisions (Fechter and Wartzack, 2017). This heuristic grasp allows for precise and predictable grab and release detection and stable movements. A more realistic approach is the multi-contract grasp. Here the target transformation is calculated from the positional change of all contact points with the hand. This would enable more natural rotations executed by small movements of the fingers but only works with stable and precise hand tracking (Boulic et al., 1996). For maximum precision, the controlled DOFs can be reduced. For example, an only translation grasp where the rotation is fixed. To detect this intent a separate gesture might be used, e.g., a pinch.
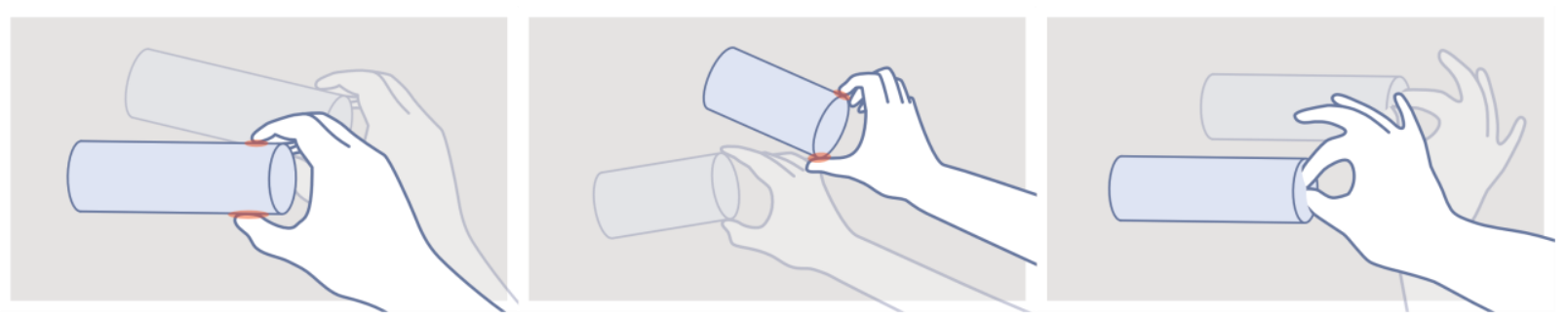

Figure 5. Body grasping interactions: a) heuristic grasp b) multi-contact grasp c) only translation grasp

\section{Pushing}

In the real world, humans often push resting objects for precise movements, instead of grasping them. This interaction can also be adapted for virtual reality. To do so, collisions of the users' fingers and hands with a virtual body are resolved by moving the touched object. Two kinds of pushing can be distinguished. On one hand, physical pushing with behavior motivated by the real-world physics could be used. Pushing a body off-center would result in a rotation because moment of inertia is simulated. This creates a natural interaction and can be combined with gravity and inertia to create a playful interface, as found in many VR games and sandboxes. On the other hand, non-physical pushing would allow for more predictable behavior. For this interaction, objects would always move away from the collision in the opposite normal direction of the touched surface point.
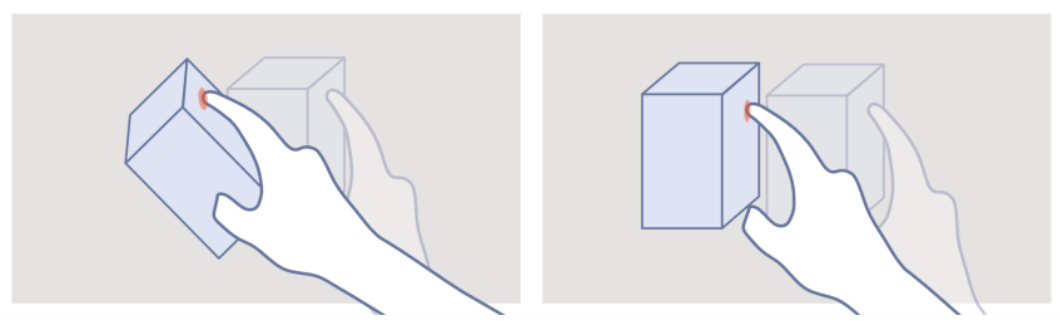

Figure 6. Body pushing interactions: a) physical pushing b) non-physical pushing

\section{Widgets}

Traditional three-dimensional geometry authoring tools make heavy use of widgets for object relocation. These are visual controls placed on the object for easy manipulation, for instance, 
Blender's transform widgets. Adding explicit controls is fundamentally unnatural. Nevertheless, the interaction can still be intuitive and allow high precision manipulation. One example of this is the smart pin (Caputo et al., 2017). Here a pin-like handle is placed on the object to be moved and points from the user's hand towards the object's center. The user can grab the handle and initiate either a rotation, a translation or a scaling. Translations are restricted to the pin's axis and optionally scaled for more precision. Rotations are more stable by the pin's leverage. An additional benefit is the unobstructed view of the manipulated object. A two-handed widget interaction for object placement is the handlebar (Song et al., 2012). When grasping with both hands in mid-air this interaction creates a bar between the hands. An object that pierced by this bar will move and rotate with it. Using positional input from two hands instead of one hand achieves very stable control over the objects' placement.

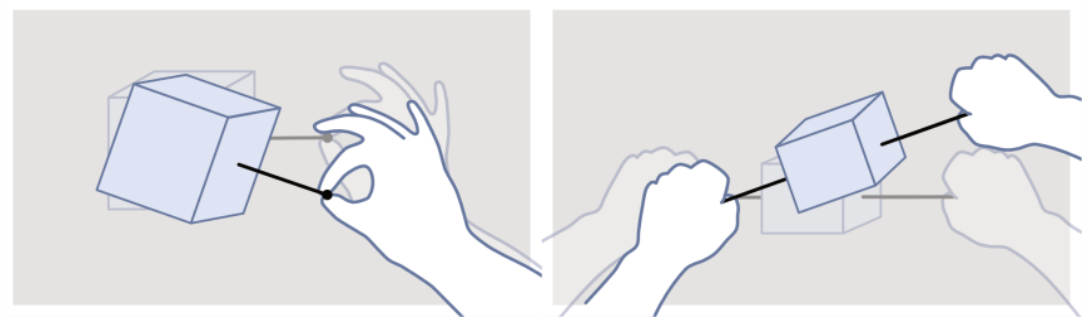

Figure 7. Widget interactions: a) smart pin b) handlebar

\subsubsection{Object selection}

Object selection is not only necessary to select operands for instantaneous operations such as deletion. It can also initiate other interactions, for example, a transformation widget. Furthermore, selection is also essential for interacting with a graphical user interface (GUI), for instance a menu. Argelaguet and Andujar (2013) collected and categorized many object selecting techniques. Three major selection categories are shown below.

An obvious way to select bodies is by touching them. It is one of the first manual interactions a human baby learns and natural for users to carry out. Implementation is simple, detecting collisions between objects and fingertips. In models of moderate complexity, it is easy for the user to touch the intended body. One drawback is the limitation to the arms reach. To select objects further away, pointing and occlusion interactions are available. Both make use of a selection ray and have theoretically infinite range. With pointing, the ray starts at a fingertip and aligns with the finger's direction. Occlusion techniques have the ray starting at the user's eyes and traveling through an occluder, typically also a fingertip. For pointing, visualizing the selection ray increases the precision, omitting it, on the other hand, could feel more natural. Both ray-based selection interactions need an additional indication when the object currently hit by the ray should be selected. A gesture, for example, touching the thumb and middle finger can be used.
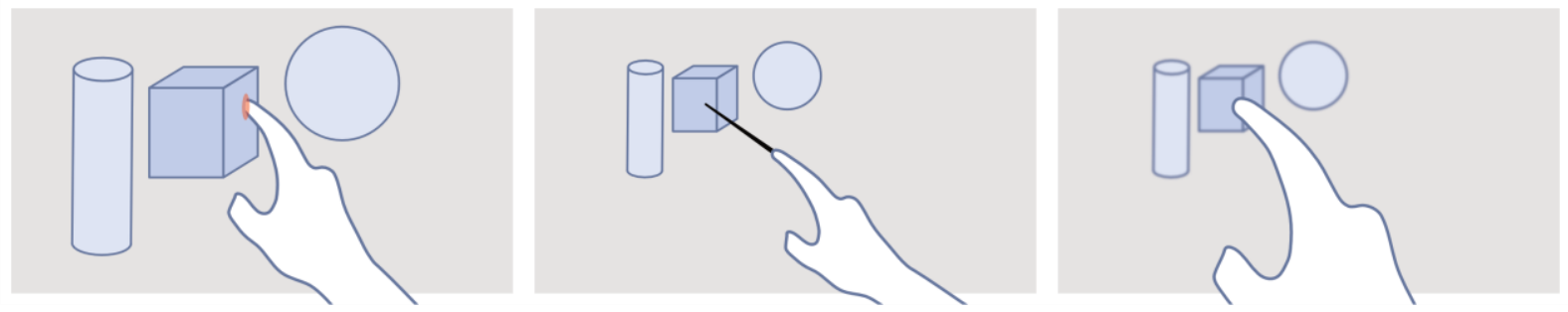

Figure 8. Object selection interactions: a) touching b) pointing c) occlusion

Some operations take multiple operands, such as grouping, Boolean combination, or bulk changes. A simple option to select more than one object is to repeatably perform the single object selection interaction. Also, cuboid selection corresponding to the widespread area selection box in traditional UX could work. Boolean operations always need two operands. For this use case, an interaction making use of the user's two hands is possible. Depending on which and in what order the bodies are moved the intended Boolean operator (union, intersection, or difference) is detected (Mendes et al., 2017). 


\subsubsection{Body related operations}

Users expect a selection of operations modifying one or multiple bodies from modeling applications. These include coloring or texturing, deletion and copying created objects. To issue such commands many barehanded interactions are possible. When designing a natural modeling system it has to be decided whether to use a uniform interaction paradigm, where all operations are chosen similarly or to use more specific interactions for each job.

Mimic gestures, used to directly describe a concept, can be used as such specific per-operation interactions (LaViola et al., 2017). While for some operations real-world counterparts exist (e.g. throwing away for deletion), finding natural mimic gestures for others (e.g. deletion) is difficult. If individual natural interactions for all interactions are found, this could create a good interface. However, if too many unintuitive gestures are necessary, the user needs to remember all of those, resulting in diminishing usability. Furthermore, extending the tool with a new feature would always result in the need for a new interaction.

One way to have a coherent interaction for operations are separate selecting objects. For example, virtual tools that can be picked up and used to issue an operation on objects selected with it. A virtual paintbrush might color objects touched with its tip. Another possibility is to assign a single operation to each fingertip, skipping the need for virtual tools.

Another way of uniform operation execution is to split the interaction into operation and operant selection. Suitable interactions for choosing the operant are found in section 3.2.2 and for choosing the operation in section 3.3 as this is a non-spatial task. The users have only two interactions to get comfortable with and it is easy to extend the system with new features. This concept is not the most natural, but very widespread in UX design and thus well known to experienced users. The order of what is selected first should be flexible, allowing the user to interact with the interface the way he is comfortable.

\subsection{Non-spatial Tasks}

Non-spatial tasks are all actions not directly related to the geometry itself. These include a wide range of operations from persistence commands such as load and save, usability improvements like undo and redo, to all kinds of general settings. As they are non-spatial, there are fewer possibilities for natural interactions for this type of task. Nevertheless, they are essential for a modeling tool and should not remain unmentioned. All already described spatial tasks could also be done with non-spatial interactions using menus for example. But the spatial versions are generally more intuitive, effective, and engaging.

\section{Symbolic gestures}

Specific symbolic gestures could be assigned to each command (LaViola et al., 2017). Undo and redo might be executed by pointing to the left and right respectively for example. This method only works with reliable gesture recognition and for a few commands. With growing complexity, the user has to remember too many gesture-command pairs and including affordances and assistance for recalling the interactions is difficult.

\section{Menus}

Adding menus contradicts the idea of a natural interface. But it is a straightforward possibility to allow the user to effectively execute non-spatial tasks. Everybody has experience with menu systems and a lot of work has been done to include them into immersive experiences as undisruptive as possible (Dachselt and Hübner, 2007).

\section{DIscussion}

This presentation of interactions shows only a section of the interface design space immersive natural modeling applications offer. Table 1 gives an overview of all presented interactions and adds a personal assessment of their naturalness, robustness against noisy tracking, implementation complexity, task completion efficiency, and functionality. Which interactions should be chosen for a specific use-case highly depends on the field of application. For an application designed for experts and daily use, efficiency might be prioritized. More natural interactions, on the other hand, can allow inexperienced users to express spatial ideas they couldn't with traditional tools. The resulting interfaces' usability not only depends on the choice of interaction modality. Many other user experience factors, such as meaningful feedback and clear affordances should be considered as well. 
When designing an application, not only the right interaction per task must be selected, but also all interactions together should form a coherent interface. Similar interaction paradigms will help the user getting comfortable. Also, not all interactions are compatible with each other, when pinching the side of a body, does the user want to move the whole object or to pull that face modifying the shape? For such collisions, different modeling modes can be introduced. Changing those is another non-spatial task and will need an interaction from that task type.

\section{Table 1. Overview of all presented tasks and interactions. Interaction properties are assessed by personal experience on a simple scale from bad (-) to good (+). Good complexity means less implementation effort.}

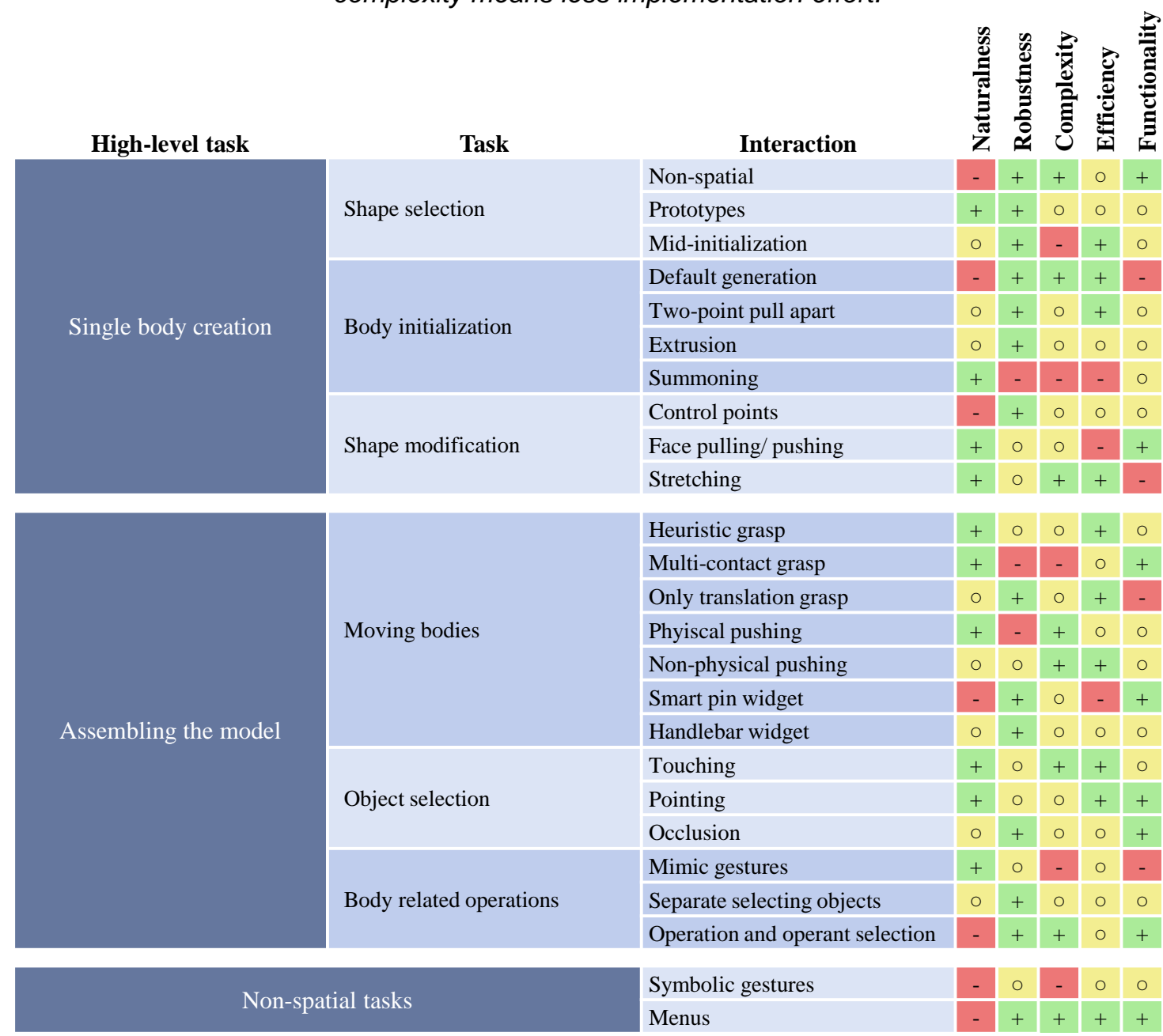

\section{CONCLUSION}

We believe natural modeling interfaces are applicable in all computer-aided engineering tasks dealing with three-dimensional geometry. But for most real-world applications the underlying technology has to improve. The biggest hurdle currently is the unstable tracking struggling with occlusion. For now, the technology seems best suited for tasks where fast modifications are more important than highest precision. Early drafts and design reviews for example. With improved hand tracking, we imagine natural modeling interactions also to be able to aid the creation of final product geometry. For use cases where long modeling sessions are to be expected, ergonomic considerations are necessary. On one hand, continuous execution of mid-air interactions might be tiring, on the other hand, these modalities could give variety to the everyday work of desk-bound product developers. A further popular application for immersive systems is remote collaborative work. For this, it is important to see and understand what the co-workers are doing. With the interactions being familiar from the real world, understanding the usage of a natural interface by someone else is easy, given a high-fidelity avatar.

No matter the field of application, the presented systematic collection of natural interactions supports researchers and developers in identifying suitable interactions for their immersive modeling systems by either implementing one of the described ones or using those as inspiration for new ones. 


\section{ACKNOWLEDGMENTS}

The authors thank the German Research Foundation for funding this research under grant number WA 2913/34-1.

\section{REFERENCES}

Argelaguet, F. and Andujar, C. (2013), "A survey of 3D object selection techniques for virtual environments", Computers \& Graphics, Vol. 37 No. 3, pp. 121-136. http://dx.doi.org/10.1016/j.cag.2012.12.003.

Borst, C.W. and Indugula, A.P. (2005), “Realistic virtual grasping”, IEEE Proceedings. VR 2005. Virtual Reality, 2005., IEEE, Bonn, Germany, pp. 91-320. http://dx.doi.org/10.1109/VR.2005.1492758.

Boulic, R., Rezzonico, S. and Thalmann, D. (1996), "Multi-finger manipulation of virtual objects", Proceedings of the ACM Symposium on Virtual Reality Software and Technology, Association for Computing Machinery, New York, NY, USA, pp. 67-74. http://dx.doi.org/10.1145/3304181.3304195.

Caputo, F.M., Emporio, M. and Giachetti, A. (2017), "The Smart Pin: A Novel Object Manipulation Technique for Immersive Virtual Environments", Proceedings of the 23rd ACM Symposium on Virtual Reality Software and Technology, ACM, New York, NY, USA, p. 89:1-89:2. http://dx.doi.org/10.1145/3139131.3141784.

Coburn, J.Q., Freeman, I. and Salmon, J.L. (2017), “A Review of the Capabilities of Current Low-Cost Virtual Reality Technology and Its Potential to Enhance the Design Process", Journal of Computing and Information Science in Engineering, Vol. 17 No. 3. http://dx.doi.org/10.1115/1.4036921.

Cordeiro, E., Giannini, F. and Monti, M. (2019), "A survey of immersive systems for shape manipulation", Computer-Aided Design and Applications, Vol. 16 No. 6, pp. 1146-1157. http://dx.doi.org/10.14733/cadaps.2019.1146-1157.

Cordeiro, E., Giannini, F., Monti, M., Mendes, D. and Ferreira, A. (2018), “A Study on Natural 3D Shape Manipulation in VR”, Smart Tools and Apps for Graphics - Eurographics Italian Chapter Conference, p. 9 pages. http://dx.doi.org/10.2312/stag.20181296.

Dachselt, R. and Hübner, A. (2007), “Three-dimensional menus: A survey and taxonomy”, Computers \& Graphics, Vol. 31 No. 1, pp. 53-65. http://dx.doi.org/10.1016/j.cag.2006.09.006.

Fechter, M., Schleich, B. and Wartzack, S. (2020), "CAD-Gestaltmodellierung in VR für die frühe Entwurfsphase", Konstruktion, Vol. 72, pp. 69-74.

Fechter, M. and Wartzack, S. (2017), "Natural Finger Interaction for CAD Assembly Modeling”, in ASME (Ed.), ASME 2017 International Design Engineering Technical Conferences and Computers and Information in Engineering Conference, Volume 1: 37th Computers and Information in Engineering Conference, p. V001T02A041. http://dx.doi.org/10.1115/DETC2017-67555.

Harlan, J., Schleich, B. and Wartzack, S. (2020), "Linking a game-engine with CAD-software to create a flexible platform for researching extended reality interfaces for the industrial design process", Proceedings of the 31st Symposium Design for X, The Design Society, pp. 169-178. http://dx.doi.org/10.35199/dfx2020.18.

Kang, J., Zhong, K., Qin, S., Wang, H. and Wright, D. (2013), “Instant 3D design concept generation and visualization by real-time hand gesture recognition”, Computers in Industry, Vol. 64 No. 7, pp. 785-797. http://dx.doi.org/10.1016/j.compind.2013.04.012.

LaViola, J.J., Kruijff, E., McMahan, R.P., Bowman, D.A. and Poupyrev, I. (2017), 3D User Interfaces: Theory and Practice, Second edition., Addison-Wesley, Boston.

Mendes, D., Medeiros, D., Sousa, M., Ferreira, R., Raposo, A., Ferreira, A. and Jorge, J. (2017), “Mid-air modeling with Boolean operations in VR”, 2017 IEEE Symposium on 3D User Interfaces (3DUI), pp. 154157. http://dx.doi.org/10.1109/3DUI.2017.7893332.

Pahl, G., Beitz, W., Schulz, H.-J. and Jarecki, U. (2013), Pahl/Beitz Konstruktionslehre: Grundlagen erfolgreicher Produktentwicklung. Methoden und Anwendung, Springer-Verlag.

Rautaray, S.S. and Agrawal, A. (2015), "Vision based hand gesture recognition for human computer interaction: a survey”, Artificial Intelligence Review, Vol. 43 No. 1, pp. 1-54. http://dx.doi.org/10.1007/s10462-012-9356-9.

Regazzoni, D., Rizzi, C. and Vitali, A. (2018), "Virtual Reality Applications: Guidelines to Design Natural User Interface", Volume 1B: 38th Computers and Information in Engineering Conference, American Society of Mechanical Engineers, Quebec City, Quebec, Canada, http://dx.doi.org/10.1115/DETC2018-85867.

Song, P., Goh, W.B., Hutama, W., Fu, C.-W. and Liu, X. (2012), “A handle bar metaphor for virtual object manipulation with mid-air interaction", Proceedings of the 2012 ACM Annual Conference on Human Factors in Computing Systems CHI '12, ACM Press, Austin, Texas, USA, p. 1297. http://dx.doi.org/10. $1145 / 2207676.2208585$.

Vuletic, T., Duffy, A., Hay, L., McTeague, C.P., Campbell, G., Choo, P.L. and Grealy, M. (2018), "Natural and intuitive gesture interaction for 3D object manipulation in conceptual design", DS 92: Proceedings of the DESIGN 2018 15th International Design Conference, pp. 103-114. http://dx.doi.org/10.21278/idc.2018.0321. 